\title{
Schlafbezogene Atmungsstörungen
}

\author{
Th. Zahnert
}

\section{Facharztwissen HNO}

Dieser 7. Beitrag einer neuen Serie von Publikationen in der Laryngo-Rhino-Otologie widmet sich dem Thema „Schlafbezogene Atmungsstörungen“. Über das Jahr verteilt erscheinen in unserer neuen Rubrik „Facharztwissen HNO“ alle 3 Monate didaktisch aufbereitete Übersichtsartikel. Diese greifen die Hauptthemen der Kursreihe „Facharztwissen HNO" auf, die im Wechsel an den HNO-Kliniken in Dresden, Erfurt, Jena und Leipzig stattfindet (jährliche Veranstaltung der Professoren Andreas Dietz, Orlando Guntinas-Lichius, Thomas Zahnert und Dirk Esser). Diese Kursreihe zielt erstrangig auf Assistenten in der Weiterbildung HNO-Heilkunde ab und soll in möglichst persönlichem Rahmen eine direkte Diskussion der Lehrinhalte im Rahmen eines interaktiven Workshops ermöglichen. Die Autoren haben sich zur Aufgabe gestellt, die Inhalte praxisgerecht mit Rücksicht auf aktuelle, wissenschaftlich belastbare Entwicklungen anzubieten. Der Inhalt des HNO-Facharztwissens unterliegt einem ständigen Fluss, sodass durch das Konsensusverfahren der 4 genannten ausgewiesenen Fachvertreter die Aktualitaet und Belastbarkeit des angebotenen Wissens angestrebt wird.

Merke: Die neue Rubrik „Facharztwissen HNO“ erscheint viermal im Jahr, ist konsequent arbeitsplatzorientiert und praxisnah, mit vielen Tipps und Tricks und konkreten Anweisungen für eine zeitgemäße Diagnostik und Therapie.

\section{Einleitung}

Längst hat die Schlafmedizin mit der Entwicklung von internationalen Standards in der Diagnostik und Therapie von Schlafstörungen einen festen Platz in der medizinischen Versorgung eingenommen. Dabei gibt es essenzielle Berührungspunkte mit der Hals-Nasen-Ohrenheilkunde - insbesondere auf dem Feld der schlafbezogenen Atmungsstörungen. Obstruktionen im Bereich der oberen Luftwege zählen zu den häufigsten Ursachen und liegen damit diagnostisch und therapeutisch im Fokus des
Hals-Nasen-Ohrenarztes. Für die richtige Therapieentscheidung sind Kenntnisse über die Grundbegriffe der Schlafmedizin und die Bewertung der modernen apparativen Diagnostikdaten unabdingbar. Insbesondere geht es dabei um das Erkennen der obstruktiven Ursache und die Abgrenzung obstruktiver Faktoren gegenüber anderen schlafbezogenen Atmungsstörungen. Nicht zuletzt zählen schlafbezogene Atmungsstörungen zu den häufigen kardiovaskulären Risiken (plötzlicher Kindstod, Schlaganfall, Myokardinfarkt, Hypertonie) und verlangen in der Regel nach einer interdisziplinären Abstimmung - insbesondere mit Kinderärzten, Kardiologen, Pulmologen und Schlafmedizinern. Der folgende Beitrag soll einen für den Facharzt für Hals-Nasen-Ohrenheilkunde relevanten Überblick über die in seinem Bereich anzutreffenden schlafbezogenen Atmungsstörungen liefern.

\section{Definition}

Schlafbezogene Atmungsstörungen (SBAS)

Ausschließlich oder primär im Schlaf auftretende

Atemstörung charakterisiert durch:

- Apnoen oder Hypopnoen mit oder ohne

pharyngeale Obstruktion

- Hypoventilationen

- Hypoxämie, Hyperkapnie, Azidose

Während unter Apnoe ein Atemstillstand verstanden wird, ist die Hypopnoe lediglich durch eine Reduktion des Atemflusses gekennzeichnet. Beide Begriffe können anhand der apparativen Schlafdiagnostik genauer beschrieben werden. Die Ereignisse müssen beispielsweise mindestens 10 Sekunden anhalten.

Schnarchen allein zählt dagegen nicht zu den schlafbezogenen Atmungsstörungen, sondern beschreibt ein akustisches Geräusch bei der Atmung im Schlaf, welches durch die Vibration von Anteilen der Pharynxmuskulatur, insbesondere des Gaumens und der Zunge, entsteht. Es ist in den meisten Fällen harmloser Natur (sog. primäres Schnarchen) und bedarf insofern keiner medizinisch indizierten Be- 
handlung. Da 95\% der Patienten mit obstruktivem Schlafapnoe-Syndrom schnarchen, wird insbesondere vor Eingriffen zur Reduktion des Schnarchens (Somnoplastik) eine schlafmedizinische Abklärung empfohlen. Die Diagnose des harmlosen primären Schnarchens lässt sich erst nach Ausschluss einer schlafbezogenen Atmungsstörung mit letzter Sicherheit stellen.

\section{Ätiologie und Einteilung}

Merke: Die Pathogenese der schlafbezogenen Atmungsstörungen ist nicht einheitlich. Grundsätzlich kann anhand der Ätiologie zwischen SBAS mit und ohne Obstruktion unterschieden werden.

In den meisten Fällen liegen die einzelnen Krankheitsbilder isoliert vor und lassen sich dann effektiv diagnostizieren. Es gibt jedoch auch obstruktive Störungen mit zentralen Komponenten und entsprechenden Herausforderungen an die Diagnostik und Therapie ( $\bullet$ Abb.1).

\section{Obstruktives Schlafapnoe- Syndrom (OSAS)}

Schlafbezogene Atmungsstörungen mit Obstruktion sind heute dem Begriff des obstruktiven Schlafapnoe-Syndroms gleichzusetzen. Ätiologisch kommt es dabei zu einer Verlegung der oberen Luftwege im Schlaf mit der Folge eines zu hohen Atemwegswiederstandes, einer vermehrten Atemanstrengung und Hypoventilation bis hin zur mechanisch erzwungenen Apnoe. Erst durch den damit verbundenen $\mathrm{CO}_{2}$-Anstieg im Blut setzt reflektorisch die Atmung wieder ein, mit vermehrter Anspannung der Atemmuskulatur und Überwinden des Atemwiderstandes. Diese Anstrengung ist allerdings mit einer Weckreaktion (Arousel) verbunden und damit mit der Redukti-

\section{obstruktive Schlafapnoe-Syndrome (OSAS)}

- bei Erwachsenen

- im Kindesalter

zentrale Schlafapnoe-Syndrome (ZSAS)

- primär zentrale Schlafapnoe

- bei Erwachsenen

- im Säuglingsalter

- ZSA bei Cheyne-Stokes-Atmung

- ZSA bei höhenbedingter periodischer Atmung

- ZSA bei anderer körperlicher Erkrankung

- ZSA bei Medikamenten/Drogen/anderen Substanzen

\section{schlafbezogene Hypoventilations-|Hypoxämie-Syndrome}

- nicht obstruktive alveoläre Hypoventilation (idiopathisch)

- kongenitales zentrales alveoläres Hypoventilationssyndrom

- durch körperliche Erkrankungen on der Schlaftiefe oder Fragmentierung des physiologischen Schlafes. Klinische Folgen sind Tagesschläfrigkeit, nachlassende Leistungsfähigkeit und eine Belastung des kardiopulmonalen Systems. Die Verlegung der Atemwege im Schlaf kann funktioneller und/oder anatomischer Natur sein.

Merke: Die häufigste Ursache im Erwachsenenalter ist der Kollaps der pharyngealen Muskulatur (einschließlich Zunge und weicher Gaumen) unter dem im Schlaf physiologisch nachlassenden Muskeltonus.

Begünstigt wird dieser Mechanismus durch Hypertrophie der Zunge, des Gaumens und der äußeren Halsweichteile bei Adipositas. Die Obstruktion kann jedoch auch im Bereich der Nase oder des Nasenrachens liegen und über eine damit erzwungene Mundatmung mit entsprechenden muskulären Folgen ebenfalls zur erschwerten Atmung beitragen. Im Kindesalter ist die Hyperplasie des Waldeyerschen Rachenringes bei adenoiden Vegetationen als häufigste Ursache des Schlafapnoe-Syndroms zu nennen [1].

Ursprünglich war in die Gruppe der obstruktiven Atmungsstörungen auch das Upper Airway Resistance Syndrom (UARS) eingeschlossen, eine Erkrankung, bei der es zu respiratorischen Ereignissen mit erhöhtem Atemwiderstand (oder respiratorischen Arousals) kommt, ohne dass Atemflussstörungen zu verzeichnen sind. Wegen der fehlenden Abgrenzbarkeit gegenüber dem OSAS wurde dieses Krankheitskonzept in den letzten Jahren wieder verlassen.

\section{Langzeitfolgen OSAS}

Die klinische Bedeutung des obstruktiven Schlafapnoe-Syndroms liegt in den langfristigen Folgen für das Herz-Kreislauf-System sowie in den Folgen des nichterholsamen Schlafes mit Tagesschläfrigkeit und Vigilanzminderung. Konkret ergeben sich daraus:

- 3-7-fach erhöhtes Unfallrisiko im Straßenverkehr

> erhöhtes Risiko für

$\rightarrow$ Hypertonie

> Herzinsuffizienz

schlaganfall

\section{Epidemiologie}

Die Prävalenz des OSAS ist vom Alter, vom Geschlecht und von individuellen Faktoren abhängig. Die Inzidenz steigt zwischen dem 35. und 65. Lebensjahr an [2]. In Studien wurden ein ApnoeHypopnoe-Index (AHI) >5/h Schlafzeit bei $9 \%$ der Frauen und 24\% der Männer im Alter von 30 bis 60 Jahren gemessen. Davon hatten jedoch nur 2-4\% eine klinische Symptomatik [3]. Bei Patienten mit Herz-Kreislauf-Erkrankungen ist die die Prävalenz um das 2-3-fache erhöht [4]. 
Zu den individuellen prädisponierenden Faktoren zählen:

- BMI - Inzidenz steigt mit zunehmenden BodyMass-Index

- kraniofaziale Besonderheiten - insbesondere Retrogenie

- Rauchen, Alkohol - besonders regelmäßiger Genuss am Abend

- Schwangerschaft

Im Kindesalter ist die Hypertrophie des Waldeyerschen Rachenringes die häufigste Ursache für ein OSAS. Andere organische Erkrankungen sind in 0 Tabelle 1 genannt.

\section{Klinisches Bild}

Als Leitsymptom zählt die Tagesschläfrigkeit. Auch das unregelmäßige Schnarchen mit Atempausen ist bei vorhandener Tagesschläfrigkeit verdächtig. Die Tagesschläfrigkeit ist dabei in der Anamnese klar abzugrenzen von der Tagesmüdigkeit.

Merke: Während die Tagesmüdigkeit lediglich eine Antriebsstörung mit häufigen Gähnen und allgemeiner Abgeschlagenheit ist, kennzeichnet die Tagesschläfrigkeit der Einschlafdrang am Tage. Tagesschläfrigkeit ist durch eine Reduktion der zentralnervösen Aktivierung (Wachheit, Daueraufmerksamkeit) und Einschlafdrang charakterisiert.

Die Tagesschläfrigkeit ist Folge des nicht erholsamen Schlafs, weist eine tageszeitliche Abhängigkeit auf und führt in monotonen Situationen mit kurzer Latenz zum Einschlafen.

Diagnostisches Stufenkonzept des obstruktiven Schlafapnoesyndroms (nach [5])

- Anamnese und klinische Untersuchung

- Abklärung von Begleiterkrankungen

- Polygrafie

- ggf. Polysomnografie

\section{Anamnese}

$\nabla$

Für die Schlafapnoe-Anamnese eignen sich am besten standardisierte Fragebögen. Für die schlafbezogenen Atmungsstörungen mit Obstruktion wird beispielsweise der „Berlin-Questionnaire“ empfohlen [4]. Daneben sind in der Anamnese besonders kardiovaskuläre Nebenerkrankungen (Hypertonie) und Genussmittel zu erfragen.
Tab. 1 Ausgewählte Krankheitsbilder mit OSAS im Kindesalter.

\begin{tabular}{|l|l|l|}
\hline mit Obstruktion & $\begin{array}{l}\text { mit Hirnstamm-I } \\
\text { Hirnnervenbeteiligung }\end{array}$ & $\begin{array}{l}\text { mit neuromuskulärer } \\
\text { Hypotonie }\end{array}$ \\
\hline adenoide Vegetationen & Achondroplasie & myotonische Dystrophie \\
\hline Tonsillenhypertrophie & Möbius-Sequenz & Prader-Willi-Syndrom \\
\hline Trisomie 21 & Myelomeningozele & Morbus Duchenne \\
\hline Mukopolysacharose & & \\
\hline Sichelzellanämie & & \\
\hline Marfan-Syndrom & & \\
\hline Goldenhar-Syndrom & & \\
\hline Alpert-Syndrom & & \\
\hline
\end{tabular}

Merke: Zu beachten ist, dass auch beim obstruktiven Schlafapnoe-Syndrom nicht immer subjektive Beschwerden angegeben werden. Oft werden Symptome wie Tagesschläfrigkeit einer hohen, aber akzeptierten beruflichen Beanspruchung zugeschrieben.

Für den HNO-Arzt stellt sich anhand der Anamnese die Frage, ob eine apparative Schlafdiagnostik im Einzelfall erfolgen sollte. Obwohl für diese Fragestellung noch keine Standards entwickelt werden konnten, spricht man vom wahrscheinlichen Vorliegen einer Schlafapnoe, der sog. PrätestWahrscheinlichkeit, wenn mehrere der folgenden Symptome vorliegen [4]:

- vermehrte Tagesschläfrigkeit

- Adipositas

- Hypertonie, überwiegend nächtliche HerzRhythmus-Störungen

- Beobachtung nächtlicher Atempausen durch den Bettpartner

- lautes, unregelmäßiges Schnarchen

- Libido- und Potenzstörungen

- unruhiger Schlaf

$\checkmark$ morgendliche Abgeschlagenheit, diffuse, dumpfe Kopfschmerzen

- Mundtrockenheit

- unspezifische psychische Symptome

- wie Abgeschlagenheit, Leistungsknick,

- Wesensänderung, intellektueller Leistungsverfall

\section{Klinische Untersuchung}

\section{$\nabla$}

Die klinische Untersuchung umfasst die Diagnostik der oberen Luftwege, d.h. die Inspektion der Nase, des Pharynx und des Larynx. Endoskopische Untersuchungen des Nasenrachens Gaumens und Zungengrundes können erste Hinweise auf anatomische Engstellen ergeben. Häufig sind ein adipöser, kurzer Hals, eine hypertrophe Zunge, die Hyperplasie der Gaumentonsillen oder der Rachenmandel sowie Kulissenphänomen und Faltenbildung des weichen Gaumens zu beobachten (OAbb. 2). 


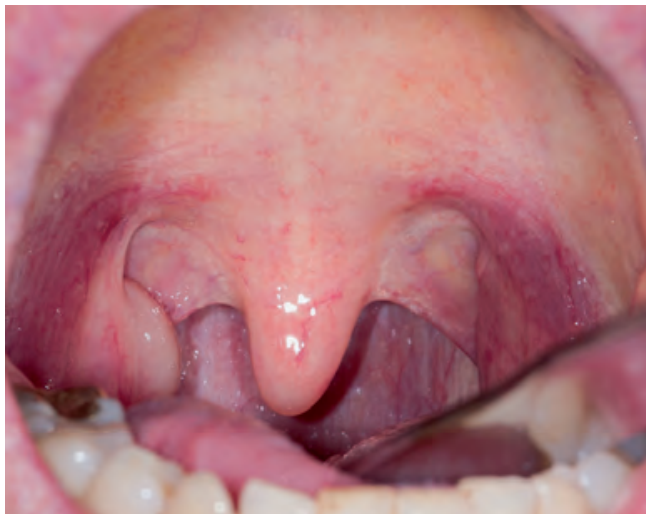

Abb. 2 Kulissenphänomen und Faltenbildung.

Abb. 3 Retrogenie im Fernröntgenbild.

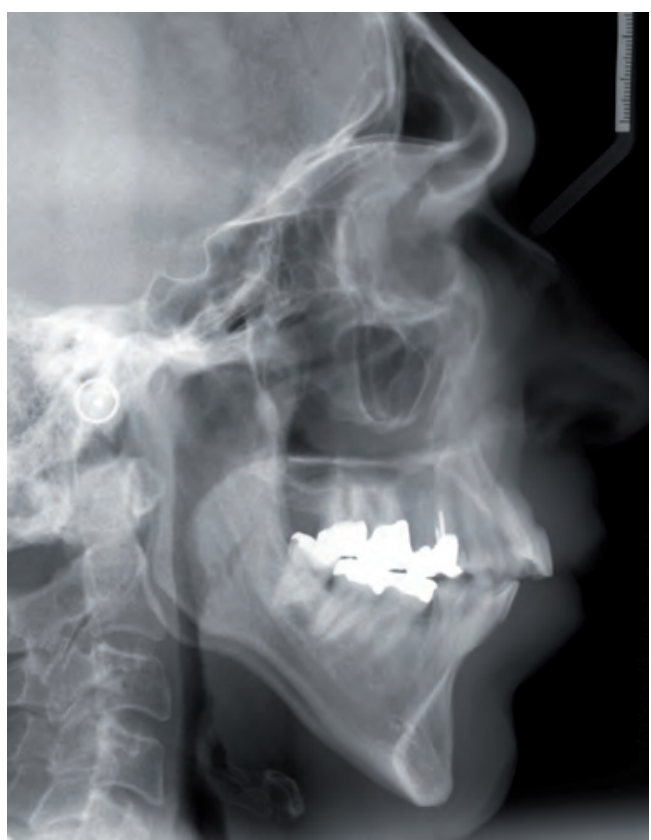

Weitere klinische Hinweise ergeben kephalometrische Untersuchungen mit metrischem Nachweis einer Retrogenie ( $\triangle$ Abb. 3) . Eine kardiopulmonale Diagnostik (EKG, Rö Thorax, RR-Messung) sollte sich anschließen.

\begin{tabular}{|c|c|}
\hline TST & Gesamtschlafzeit (Total Sleep Time) \\
\hline Apnoen/TST & Anzahl der Apnoen pro Gesamtschlafzeit \\
\hline Hypopnoen/TST & $\begin{array}{l}\text { Anzahl der Hypopnoen pro } \\
\text { Gesamtschlafzeit }\end{array}$ \\
\hline Apnoe-Index, Hypopnoe-Index & $\begin{array}{l}\text { Anzahl der Apnoen/Hypopnoen pro } \\
\text { Stunde }\end{array}$ \\
\hline Apnoe-Hypopnoe-Index (AHI) & $\begin{array}{l}\text { durchschnittliche Gesamtzahl aller Apnoen } \\
\text { und Hypopnoen pro Stunde }\end{array}$ \\
\hline Respiratory Effort Related Arousal (RERA) & $\begin{array}{l}\text { Gesamtzahl aller respiratorisch induzierten } \\
\text { Weckreaktionen pro Gesamtschlafzeit }\end{array}$ \\
\hline RERA-Index & $\begin{array}{l}\text { durchschnittliche Anzahl der RERA } \\
\text { pro Stunde }\end{array}$ \\
\hline Sauerstoffsättigung & $\begin{array}{l}\text { Mittelwert der } \mathrm{HbO}_{2} \text {-Konzentration ohne } \\
\text { Entsättigung während der TST }\end{array}$ \\
\hline Sauerstoffsättigung > 3\% & Gesamtzahl der Ereignisse pro TST \\
\hline RDI - Respiratory Disturbance Index & $\begin{array}{l}\text { Anzahl der Apnoen/Hypopnoen pro Stunde } \\
\text { Schlaf }\end{array}$ \\
\hline
\end{tabular}

\section{Apparative Schlafdiagnostik \\ A}

Für die apparative Schlafdiagnostik stehen 2 Verfahren zur Verfügung, die Polygrafie und die Polysomnografie. Die Polysomnografie wird als Goldstandard in der schlafmedizinischen Diagnostik angesehen, erfordert jedoch den Aufenthalt in einem Schlaflabor. Deshalb steht zur Erhöhung der Prätestwahrscheinlichkeit die ambulant durchführbare Polygrafie zur Verfügung, deren Zuverlässigkeit jedoch begrenzt ist.

Typische schlafmedizinisch relevante apparative gewonnene Kenngrößen sind in o Tabelle $\mathbf{2}$ zusammengestell.

\section{Polygrafie}

Die Polygrafie ist eine vereinfachte, portable Form der ausführlichen apparativen schlafmedizinischen Diagnostik ( $\bullet$ Abb. 4). Sie zeichnet respiratorische, kardiovaskuläre und akustische Parameter während des Schlafes auf, verzichtet jedoch auf die Erfassung von EEG-Signalen ( 0 Abb. 5). Sie ist für die Diagnostik der Schlafapnoe einsetzbar, zeigte jedoch in der Vergangenheit bis zu 17\% falsch negative und in bis zu $31 \%$ falsch positive Ergebnisse [6]. Durch entsprechende schlafmedizinische Schulung des Personals und Selektion der Patienten kann mit den heutigen modernen Geräten auch eine valide Diagnose bei obstruktiver Schlafapnoe getroffen werden, wenngleich zentrale Komponenten mit dieser Methode nicht erfasst werden können.

\section{Kontraindikationen}

Für folgende Fälle ist die Polygrafie nicht geeignet:

- bei fortgeschrittenen pulmonalen Erkrankungen

- bei kardiovaskulären, psychiatrischen, neurologischen Erkrankungen

- bei neuromuskulären Erkrankungen

- bei gleichzeitigen Vorliegen anderer Schlafstörungen wie der zentrale Schlafapnoe

- bei PLMD (Periodic Limb Movements in Sleep), Insomnie, zirkadianen Schlaf-Wach-Rhythmusstörungen, Narkolepsie

Notwendige Ableitungen sind:

- Atemfluss (Thermistor, Staudrucksensor)

- Atmungsanstrengung (Induktionsplethysmografie)

- Sauerstoffsättigung (Pulsoxymetrie)

- Pulsfrequenz/Körperlage

In der Regel werden zusätzlich in einem Hypnogramm die Schlafstadien, die Lautstärke des Schnarchens sowie die Weckreaktionen über die Gesamtschlafzeit (Zeitaufzeichnung) statistisch erfasst. 


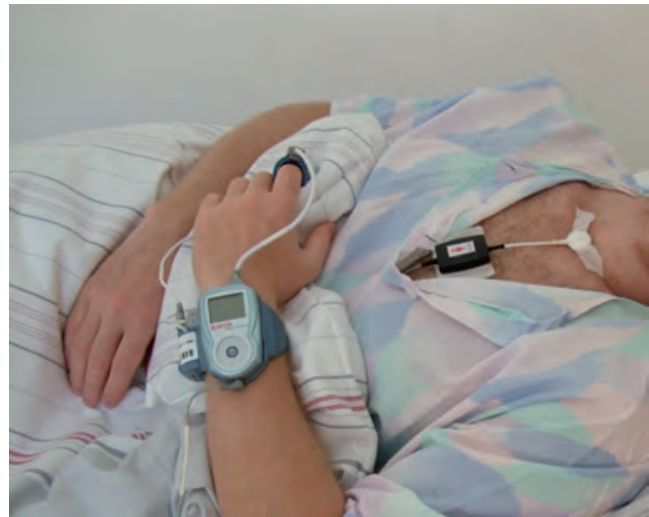

Abb. 4 Polygrafiegerät zur häuslichen Schlafdiagnostik

Polysomnografie

Die Polysomnografie wird beim obstruktiven Schlafapnoe-Syndrom zur

- Sicherung der Diagnose

- Abgrenzung gegenüber der zentralen Schlafpnoe

- Bestimmung des Schweregrades

- Anpassung der nasalen Beatmungstherapie (Atemmaske) und

- Therapieüberwachung eingesetzt.

Sie wird in schlafmedizinischen Zentren von geschultem Personal (Schlaflabor) durchgeführt $(\bullet$ Abb. 6, 7). Neben den in der Polygrafie verwendeten Kenngrößen erfasst sie zusätzlich nach den aktuellen Empfehlungen der Amerikanischen Gesellschaft für Schlafmedizin (AASM) die Aufzeichnungen von

- Schlaf-EEG - Elektroenzephalogramm

- EOG - Elektrookulogramm vom linken und rechten Orbitarand

- EMG - Elekromyogramm vom M. mentalis, M. submentalis, $M$. tibialis anterior

- EKG - mit mindestens einkanaliger Ableitung

- Videometrie.
Tab. 3 Schweregradeinteilung der OSA anhand des AHI.

\begin{tabular}{ll}
\hline leicht & $\mathrm{AHI}<15$ \\
\hline mittelgradig & $\mathrm{AHI}>15-30$ \\
\hline hochgradig & $\mathrm{AHI}>30$
\end{tabular}

Tab. 4 Schwergradeinteilung nach klinischen Symptomen.

\begin{tabular}{|l|l}
\hline leicht & $\begin{array}{l}\text { Tagesschläfrigkeit oder Einschlafzwang bei Tätigkeiten mit } \\
\text { geringer Aufmerksamkeit (Fernsehen, Lesen) } \\
\text { Tagesschläfrigkeit oder Einschlafzwang während Tätigkeiten, } \\
\text { die Aufmerksamkeit verlangen (z.B. Besprechungen) }\end{array}$ \\
\hline mittel & $\begin{array}{l}\text { Tagesschläfrigkeit oder Einschlafzwang bei Tätigkeiten, die } \\
\text { hohe Aufmerksamkeit verlangen (z.B. Autofahren) }\end{array}$
\end{tabular}

\begin{tabular}{|c|c|c|c|}
\hline \multicolumn{4}{|c|}{ Sleep Summary } \\
\hline \multicolumn{2}{|c|}{ Start Study nime: } & \multicolumn{2}{|r|}{ 10.34.19 PM } \\
\hline \multicolumn{2}{|c|}{$\begin{array}{l}\text { End Study Tme: } \\
\text { Total Study Time: }\end{array}$} & \multicolumn{2}{|r|}{ 05:47.41 AM } \\
\hline \multicolumn{2}{|c|}{ Total Study Time: } & \multicolumn{2}{|r|}{$7 \mathrm{hrs}, 13 \mathrm{~min}$} \\
\hline \multicolumn{2}{|c|}{ Sleep Time } & \multicolumn{2}{|r|}{6 hrs, 22 min } \\
\hline \multicolumn{2}{|c|}{ * REM of Sleep Time: } & & 22.4 \\
\hline \multicolumn{4}{|c|}{ Respiratory Indices } \\
\hline & REM & NREM & All Night \\
\hline PRDE: & 35.6 & 7.5 & 13.8 \\
\hline pAHI: & 27.3 & 42 & 9.4 \\
\hline ODt: & 14.7 & 1.4 & 4.4 \\
\hline
\end{tabular}

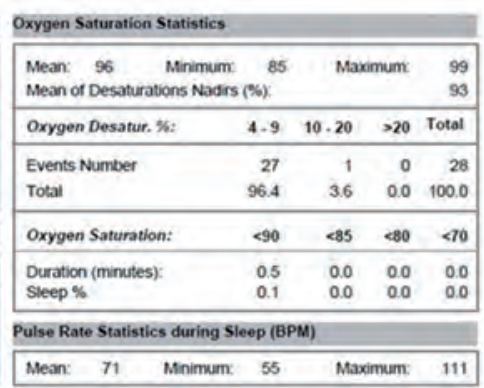

indices are calculated using vald seeep time of $6 \mathrm{hrs}, 22$ min
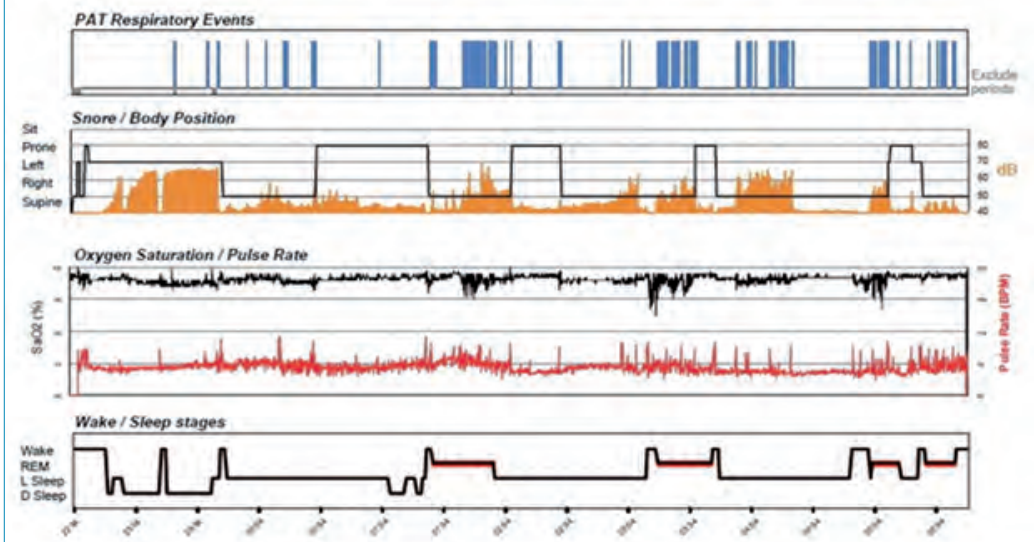

Abb. 5 Typische Polygrafieaufzeichnung

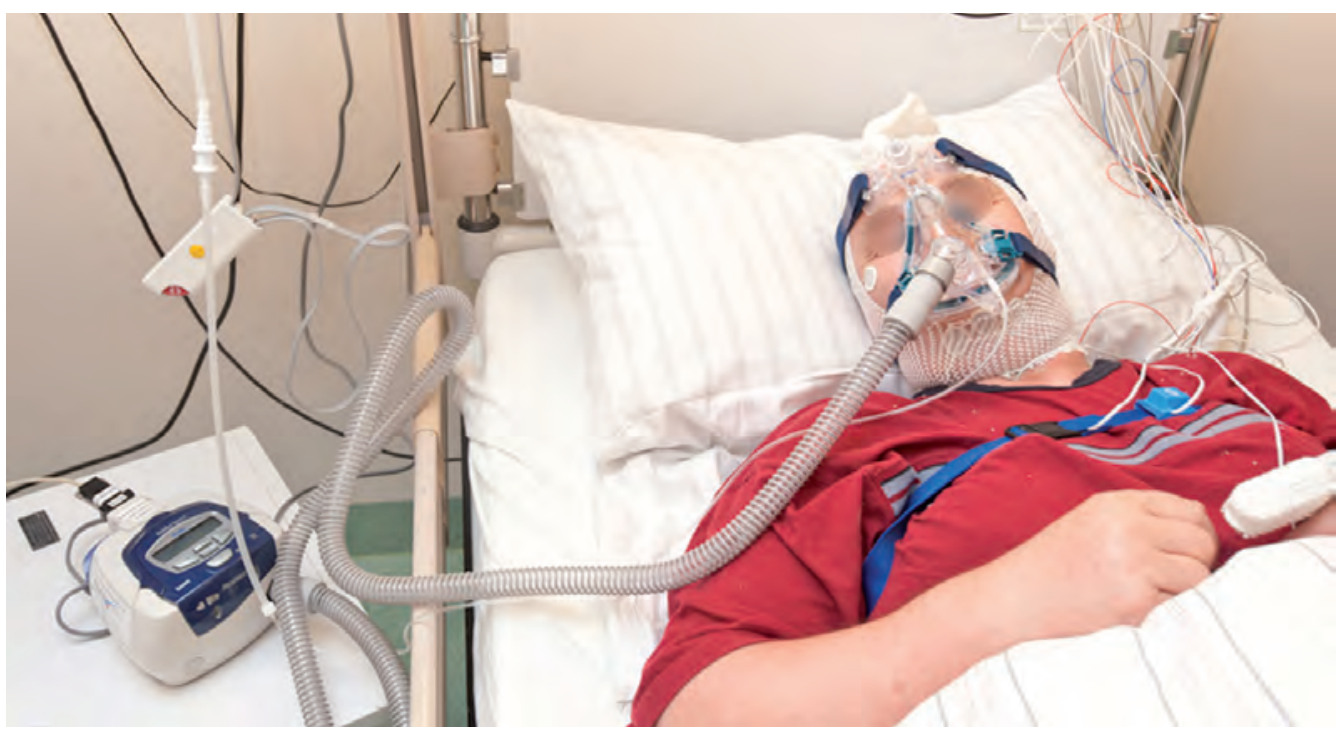

Abb. 6 Polysomnografie. 
Abb. 7 Polysomnografieaufzeichnung.
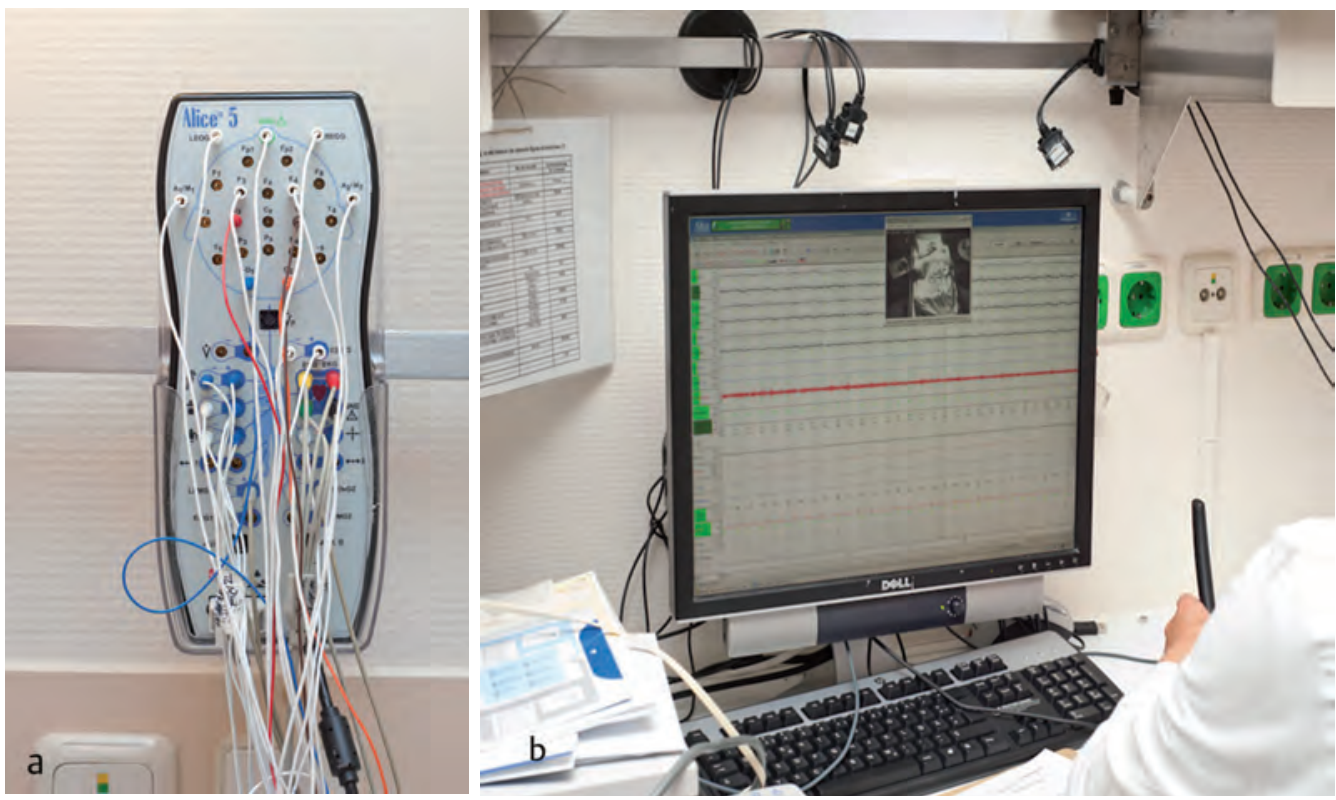

Diagnosestellung

Nach den Richtlinien der AASM kann die Diagnose eines obstruktiven Schlafapnoe-Syndroms aus der Kombination bestimmter klinischer und polysomnografischer Kriterien gestellt werden (o Abb. 8).

Merke: Entsprechend der ICSD-2 wird eine obstruktive Schlafapnoe (OSA) dann diagnostiziert, wenn die Atmungsstörung durch keine andere Schlafstörung oder medizinische Erkrankung oder durch Medikamente oder andere Substanzen erklärbar ist und entweder ein AHI>15/ h Schlafzeit oder ein $\mathrm{AHI} \geq 5 / \mathrm{h}$ Schlafzeit in Kombination mit einer typischen klinischen Symptomatik vorliegt [4].

Liegen in der Polysomnografie mehr als 15 respiratorische Ereignisse vor, gilt die Diagnose auch ohne positive Anamnese als gesichert.

Einteilung nach dem Schweregrad

Anhand des Apnoe-Hypopnoe-Index kann der Schweregrad der obstruktiven Schlafapnoe abgeschätzt werden ( $\bullet$ Tab. 3). Einige Autoren kritisie-

\begin{tabular}{|c|}
\hline \multicolumn{1}{|c|}{$\begin{array}{c}\text { positive } \\
\text { Anamnese }\end{array}$} \\
\hline - Einschlafzwang bei \\
- Tagesschläfrigkeit \\
- Schlaflosigkeit \\
- nichterholsamer \\
Schlaf \\
- nächtliches Erwachen \\
nach Atempausen mit \\
Luftnot \\
- Schnarchen mit Atem- \\
pausen
\end{tabular}

\begin{tabular}{|l|}
\hline \multicolumn{1}{|c|}{ positive } \\
Polysomnographie \\
\hline - 5 oder mehr respira- \\
torische Ereignisse \\
(Apnoe, Hypopnoe, \\
RERAs) pro Stunde \\
Schlaf \\
erhöhte Atemarbeit \\
\hline
\end{tabular}

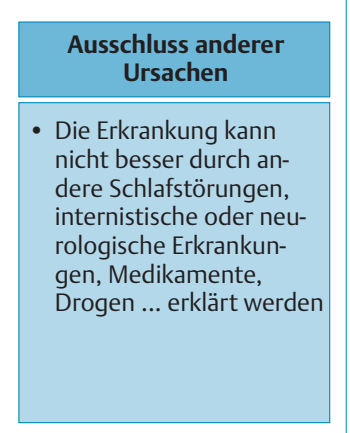

Abb. 8 Diagnosekriterien OSA ren jedoch die Nichtbeachtung der klinischen Symptomatik bei diesem Vorgehen.

Der Schweregrad der obstruktiven Schlafapnoe korreliert in der Regel mit einer typischen klinischen Symptomatik ( Tab. 4).

\section{Therapie des obstruktiven Schlafapnoe-Syndroms}

Für die Therapie stehen konservative, apparative und operative Methoden zur Verfügung. Die Auswahl des richtigen Therapieverfahrens richtet sich nach der Ursache der Obstruktion, dem Schweregrad und individuellen Faktoren ( $\triangle$ Abb. 9).

\section{Konservative Therapie \\ $\nabla$}

Zu den konservativen Methoden zählen

- Schlafhygiene,

- Schlafpositionierung,

- Gewichtsreduktion.

In früheren Behandlungsstrategien waren zusätzlich medikamentöse Ansätze vertreten, diese sind anhand der Studienlage jedoch verlassen worden.

\section{Schlafhygiene}

Zu den grundlegenden Voraussetzungen eines erholsamen Schlafes zählt die Einhaltung einer individuellen Schlafdauer, die beim Erwachsenen zwischen 4 und 12 Stunden stark variieren kann. In dieser Zeit werden in der Regel 4-7 Schlafzyklen durchlaufen. Störungen des Schlafrhythmus 


\section{OSA Therapie}

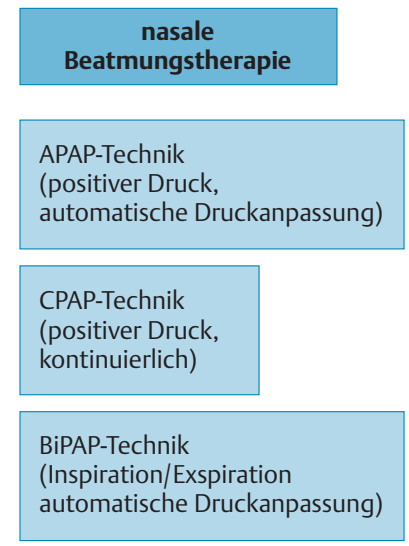

\begin{tabular}{c|c|}
\hline konservative \\
Therapie
\end{tabular}

Verhaltenstherapie

- Gewichtsreduktion

- Schlafhygiene

- Alkoholl

Medikamentenkarenz

Hilfsmittel
- Protrusionsschiene
- Nasenklammer
- Lagerungshilfen

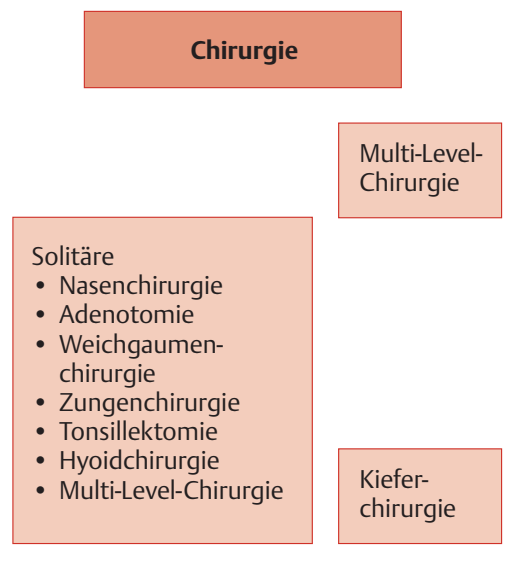

Abb. 9 Synopsis der OSA-Therapie. können durch Verhaltensmaßnahmen vermieden werden (siehe Infobox). Regelmäßiger Alkoholgenuss (Muskeltonus) sowie die späte Einnahme opulenter Mahlzeiten stören den Schlafrhythmus. Außerdem sollten Sedativa vermieden werden.

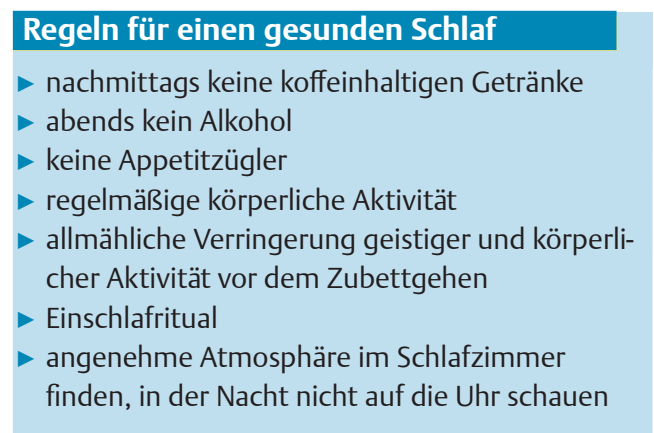

\section{Schlafposition}

Die Polygrafie und Polysomnografie erfasst auch die Lageabhängigkeit bei OSAS. Häufig treten durch die zurückfallende Zunge, der Tonsillen und des weichen Gaumens besonders in Rückenlage Obstruktionen auf. Zur Vermeidung der Rückenlage haben sich Polsterungen des Rückens bewährt (Schlafweste, Schlafrucksack) mit einer durchschnittlichen Erfolgsquote um 68\%, allerdings deutlich eingeschränkter Langzeit-Compliance ( Abb. 10) [7].

\section{Gewichtsreduktion}

Übergewicht ist ein Hauptrisikofaktor für die obstruktive Schlafapnoe. Durch die Beteiligung des bei Adipositas hypertrophen Zungengrundes und der auf den Pharynxschlauch in Rückenlage drückenden Halsweichteile erscheint die Gewichtsreduktion eine logische Konsequenz im Therapieansatz. Leider sind die Erfolgsaussichten nur moderat. Einerseits konnte belegt werden, dass eine Gewichtsreduktion um 10-15\% zu einer mittelfristigen Reduktion des AHI um 50\% führt [2].

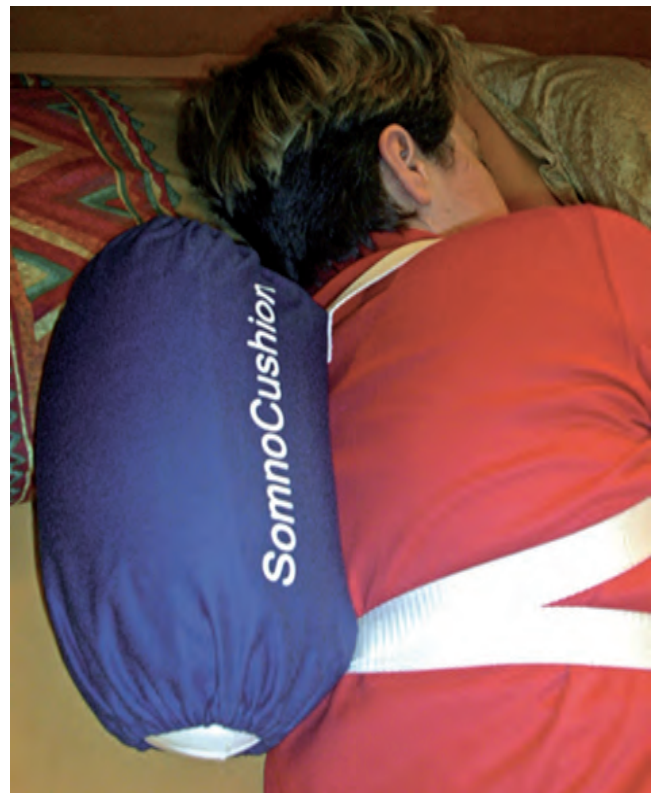

Abb. 10 Schlafrucksack.

Langfristige Beobachtungen zeigen jedoch, dass die Gewichtsreduktion nur in wenigen Fällen dauerhaft gehalten werden kann und selbst bei Patienten mit dauerhafter Reduktion sich oft eine obstruktive Schlafapnoe langfristig wieder einstellt. Dennoch wird grundsätzlich eine Gewichtsreduktion empfohlen, weil sie auch die apparative und operative Therapie günstig beeinflusst [7].

Merke: Gewichtsreduktion sollte bei Adipositas immer Teil der OSA-Therapie sein. Als alleinige Behandlungsmaßnahme kann die Gewichtsreduktion nur bei einer leichtgradigen OSA empfohlen werden.

\section{Apparative Therapie \\ $\nabla$}

Die apparative Therapie unterscheidet zwischen Hilfsmittelanwendungen zur Offenhaltung des Luftweges und der nasalen Beatmungstherapie. Andere Therapieversuche, wie z.B. die Elektrosti- 


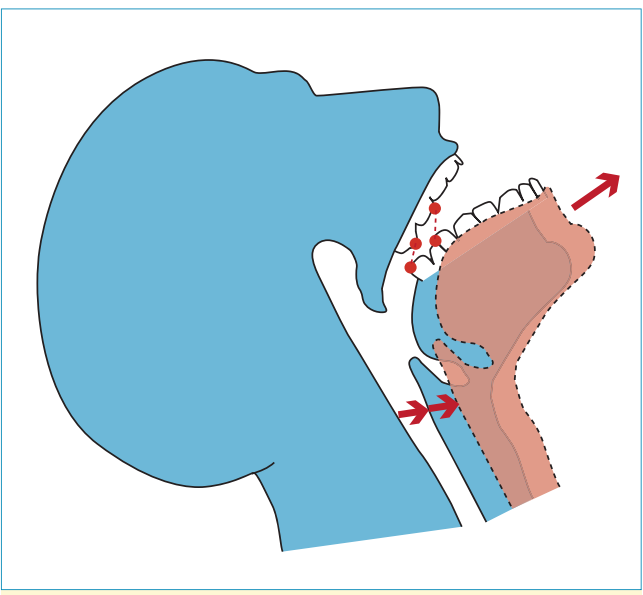

Abb. 11 Funktionsprinzip einer Protrusionsschiene zur Erweiterung des Zungengrundes bei OSAS.

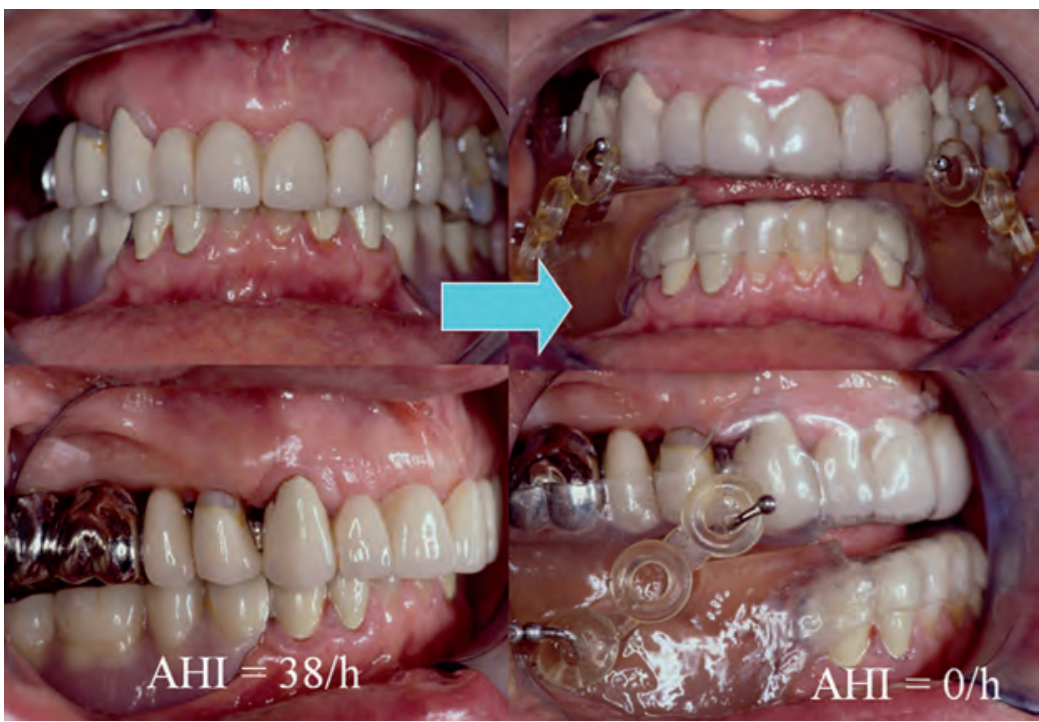

Abb. 12 Apnoe-Hypopnoe-Index vor und nach Protrusionsschienenbehandlung (Foto der Klinik für Kieferorthopädie, Universitätsklinikum Dresden, Prof Harzer)

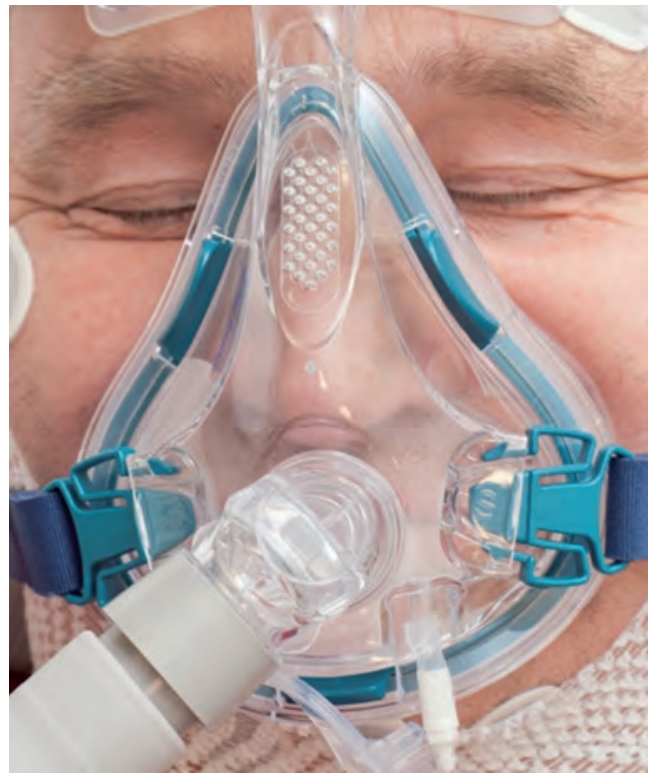

Abb. 13 Atemmaske zur nasalen Beatmungstherapie. mulation der Mundbodenmuskulatur, sind bisher ohne EBM-Empfehlungsgrad.

\section{Hilfsmittel}

Nasale Dilatatoren. Bei behinderter Nasenatmung infolge einer Instabilität des Nasenvorhofes oder der Nasenklappe erscheinen interne oder externe nasale Dilatatoren geeignet, die Obstruktion während des Schlafes zu beseitigen. Während die Wirkung als alleinige Behandlungsmaßnahme anhand von Studien bisher nicht nachgewiesen wurde, können die Dilatatoren zur Unterstützung der nasalen Beatmungstherapie empfohlen werden [7].

Unterkieferprotrusionsschiene. Unter den konservativen Maßnahmen wird die Behandlung mit einer Unterkieferprotrusionsschiene als die Maßnahme mit der höchsten Erfolgsquote (bis zu 70\%) betrachtet ( $\triangle$ Abb. 11, 12) [7]. Besonders geeignet scheint die Schienenanwendung bei

- Retrogenie,

- Überwiegen der Zungengrundobstruktion,

- rückenlageassozierter OSAS,

- guter Toleranz (Würgereiz).

Grundsätzlich wird die Schienenbehandlung für milde bis mittlere Schweregrade empfohlen. Nebenwirkungen sind vor allem Kiefergelenkprobleme, Schleimhautdruckstellen und Zahnfehlstellungen [8]. Die langfristige Compliance der alleinigen Schienenbehandlung ist deshalb unsicher und wird zwischen 40 und $80 \%$ angegeben [9].

\section{Nasale Beatmungstherapie}

Merke: Die nasale Beatmungstherapie ist mit einer Erfolgsrate von 98\% die erfolgsreichste Methode zur Behandlung der OSA und ist damit von der Wirkung mit einer Tracheotomie vergleichbar.

Sie erfolgt als kontinuierliche Überdruckbeatmung (CPAP) am spontan atmenden Patienten und gewährleistet über den Druckaufbau ein Offenhalten der Atemwege bis zur Trachea (pneumatische Schienung). Technisch erfolgt die Beatmung über eine Atemmaske, welche den Druck über die Nase appliziert ( 0 Abb. 13). Dabei kann zwischen zwei verschiedenen Techniken der Druckregulation unterschieden werden.

\section{CPAP-Technik}

Klassische Technik. Es erfolgt eine kontinuierliche Beatmung mit positivem Atemwegsdruck (3-20 mbar) und gleichen Druckverhältnissen bei Inund Expiration. Moderne Geräte verfügen über eine langsame Zunahme des Druckes in einer voreingestellten Zeit, um das Einschlafen zu erleichtern (Rampenfunktion). 


\section{Vorteile}

Die Erfolge der nasalen Beatmungstherapie konnte durch zahlreiche Studien belegt werden. Dazu zählen:

> vollständige Beseitigung der Apnoen/Hypopnoen

- Verbesserung des Schlafrhythmus

- Verbesserung der Lebensqualität (Reduktion der Tagesschläfrigkeit, Verbesserung der kognitiven Leistungen)

- Verbesserung der Lebenserwartung durch Reduktion des kardiovaskulären Risikos

(z.B. Reduktion der Hypertonie um 7-10mmHg,

Reduktion von Arteriosklerose, Reduktion des Schlaganfallrisikos)

- Reduktion des Risikos für Verkehrsunfälle

\section{Nachteile}

- Vor allem die eingeschränkte Maskentoleranz. Je jünger die Patienten sind und je geringgradiger die Schlafapnoe mit entsprechend moderaten Einschränkungen der Lebensqualität, desto schlechter ist die Toleranz. Ein Body-Mass-Index (BMI) unter $30 \mathrm{~kg} / \mathrm{m}^{2}$, ein Apnoe-Hypopnoe-Index (AHI) unter 30/h, geringe Tagesschläfrigkeit und hohe CPAP-Drücke verschlechtern die CPAP-Compliance [7]. Darüber hinaus liegt auch die Langzeittoleranz bei älteren Patienten nur bei 70\% [10]. Folglich bevorzugen Patienten mit geringgradidem SAS die Anwendung der Protrusionsschiene oder eine operative Behandlung. Die nasale CPAP-Therapie ist deshalb der Goldstandard für die Behandlung des mittel- bis schwergradigen obstruktiven Schlafapnoe-Syndroms.

\section{APAP- (Automatic Position Airway Pressure-)Tech-} nik. Der Beatmungsdruck wird automatisch der wechselnden individuellen Situation angepasst. Beispielsweise kann sich der notwendige Druck bei Wechsel aus der Rücken- in die Seitenlage ändern und durch Erfassen des Atemdruckes automatisch angepasst werden.

BiPAP-Techniken. Inspiration und Expiration werden mit verschiedenen Beatmungsdrücken eingestellt bzw. automatisch angepasst. Durch einen niedrigeren expiratorischen Druck kann die Ausatmung erleichtert und damit die Spontanatmung unterstützt werden.

Anatomische oder funktionelle Engstellen in der Nase erfordern einen höheren Druckaufbau. Da die Maskentoleranz mit zunehmenden Beatmungsdruck sinkt, ist vor jeder Maskenanpassung eine hals- nasen- ohrenärztliche Untersuchung und ggf. die operative Korrektur der nasalen Engstellen (z.B. Septumplastik, Chirurgie der Nasenmuscheln) gefordert. Die Anpassung der Atemmaske sollte in einem Schlaflabor in der Regel an 2 aufeinanderfolgenden Nächten erfolgen.

\section{Operative Therapie}

$\nabla$

Operationen an Engstellen der oberen Luftwege nehmen im Behandlungskonzept der obstruktiven Schlafapnoe in Kombination mit der Protrusionsschienenbehandlung oder Maskenbeatmung oder auch als alleinige Maßnahme einen festen Platz ein. Bei der Indikationsstellung müssen jedoch die Erfolgschancen kritisch beurteilt und insbesondere der apparativen Therapie gegenüber gestellt werden.
Cave: Wenig erfolgversprechend ist die operative Behandlung als alleinige Therapie bei:

- Adipositas per magna; $\mathrm{BMI}>30 \mathrm{~kg} / \mathrm{m}^{2}$

$-\mathrm{AHI}>25$

- kardiopulmonalen/vaskulären Nebenerkran-

kungen.

Eingriffe an der Nase und im Nasopharynx Eingriffe zur Verbesserung der Nasenatmung sind dem HNO-Arzt vertraut und werden anhand der äußeren Inspektion, der Rhinoskopie und der Rhinomanometrie indiziert. Die Eingriffe umfassen Korrekturen des Septums (bei Septumdeviation), der knöchernen Nase (Spornbildung, Schiefnase, Spannungsnase sowie der Nasenspitze und $\mathrm{Na}$ senklappe). Korrekturen der Nasenatmung werden bei entsprechender Behinderung vor allem empfohlen, um die Maskenbeatmung zu erleichtern (Reduktion des Beatmungsdruckes). Als alleinige Methode zur Behandlung des OSA erscheinen sie jedoch nicht geeignet. Bei leichtgradiger Betroffenheit können Korrekturen der Nase in Verbindung mit Korrekturen des Gaumens und Zungengrundes ebenfalls erfolgversprechend sein (s. Multi-Level-Chirurgie).

Merke: Im Nasopharynx sind es vor allem die adenoiden Vegetationen, die zusammen mit der Hypertrophie der Gaumenmandel 70-75\% der OSA im Kindesalter verursachen [11]. Als Therapie der Wahl kommt die Adenotomie ggf. in Kombination mit der Tonsillotomie/Tonsillektomie in Frage.

Beim Erwachsenen sind gutartige Obstruktionen im Nasopharynx selten in Zusammenhang mit einer chronischen Rhinosinusitis und entsprechender Polyposis oder Hyperplasie der Schleimhaut anzutreffen. 


\section{Eingriffe am weichen Gaumen und den Tonsillen}

Die Tonsillenhypertrophie kann wesentlich zur Obstruktion des oropharyngealen Isthmus und damit zum OSA beitragen. Als substanzielle Tonsillenhyperplasie und Indikation zur Operation bei OSA wird beim Erwachsenen ein intertonsilllärer Restspalt von $5 \mathrm{~mm}$ bei geöffnetem Mund betrachtet [12]. Die Tonsillotomie oder Tonsillektomie hat auch im Erwachsenenalter eine hohe Erfolgsrate von bis zu $80 \%$ zur Behandlung des OSA. Laut Leitlinie ist bei nicht tonsillektomierten Patienten, bei denen eine chirurgische Behandlung der OSA erfolgen soll, die Tonsillektomie indiziert (EBM-Empfehlungsgrad C) [7].

Merke: Bei einer substanziellen Tonsillenhypertrophie kann die alleinige Tonsillektomie dem Patienten unabhängig vom $\mathrm{AHI}$ und $\mathrm{BMI}$ empfohlen werden, da in der Mehrzahl der Fälle eine Beseitigung, zumindest jedoch eine klinisch relevante Besserung der OSA erzielt werden kann.

Der weiche Gaumen ist nicht nur die häufigste Ursache für Schnarchgeräusche, sondern kann über Steifigkeitsverlust und Überlänge im Schlaf wie ein Vorhang zur Obstruktion beitragen. Für die Chirurgie des weichen Gaumens stehen verschiedene Methoden zur Verfügung. Grundsätzlich kann zwischen resektiven Verfahren und Verfahren zur Stabilisierung des weichen Gaumens unterschieden werden. Insgesamt haben sich in den letzten Jahren funktionserhaltende Methoden gegenüber radikalen Methoden durchgesetzt. Stabilisierungen des weichen Gaumens können durch gezielte interstitielle Narbensetzungen (z.B. durch Radiofrequenztherapie) erfolgen, haben aber ihre Hauptindikation bei der Behandlung des Schnarchens. Weichgaumenimplantate aus Polyethylenterephthalat sind bisher an wenigen Patienten auch bei OSAS eingesetzt worden und werden aufgrund ihrer minimalen Invasivität empfohlen, obwohl der Effekt auf den AHI als moderat eingeschätzt wird [7]. Als resektives Verfahren ist heute die UPPP (Uvulopalatopharyngoplastik) etabliert, die bei tief hängendem Gaumensegel in Frage kommt und heute die Uvula aussparen sollte (O Abb. 14). Dabei wird überschüssige Schleimhaut reseziert, ohne die Muskulatur des Gaumens zu verletzen. Durch gezielte Nähte wird anschließend der Gaumen ausgeformt. Die UPPP kann insbesondere bei gleichzeitig durchgeführter Tonsillektomie zur Behandlung des OSAS empfohlen werden, wenn konservative Maßnahmen ausgeschöpft sind. Die Gaumenplastik kann auch resektiv, aber ohne plastische Nahtsetzung mit dem Laser durchgeführt werden (LAUP-Laser Assisted Uvula Palatoplasty). Aufgrund der dabei nicht kontrollierbaren narbigen Verziehungen des Gaumens wird die Methode zur Behandlung des OSAS als kontraindiziert betrachtet [7].

\section{Eingriffe an der Zunge und am Zungen- grund}

Die Hypertrophie der Zunge und insbesondere des Zungengrundes zählt zu den am schwierigsten zu behandelnden Ursachen der OSA. Resektive Verfahren haben hier durch ein hohe Rezidiv- und Nebenwirkungsrate nur eingeschränkte Indikationen.

Radiofrequenztherapie des Zungengrundes. Sie kann als minimal invasives Verfahren gezählt werden (in LA und ambulant durchführbar).

Merke: Bei leichten und mittelgradigen OSA liegt die Kurzzeiterfolgsrate nach Radiofrequenztherapie bei $33 \%$.
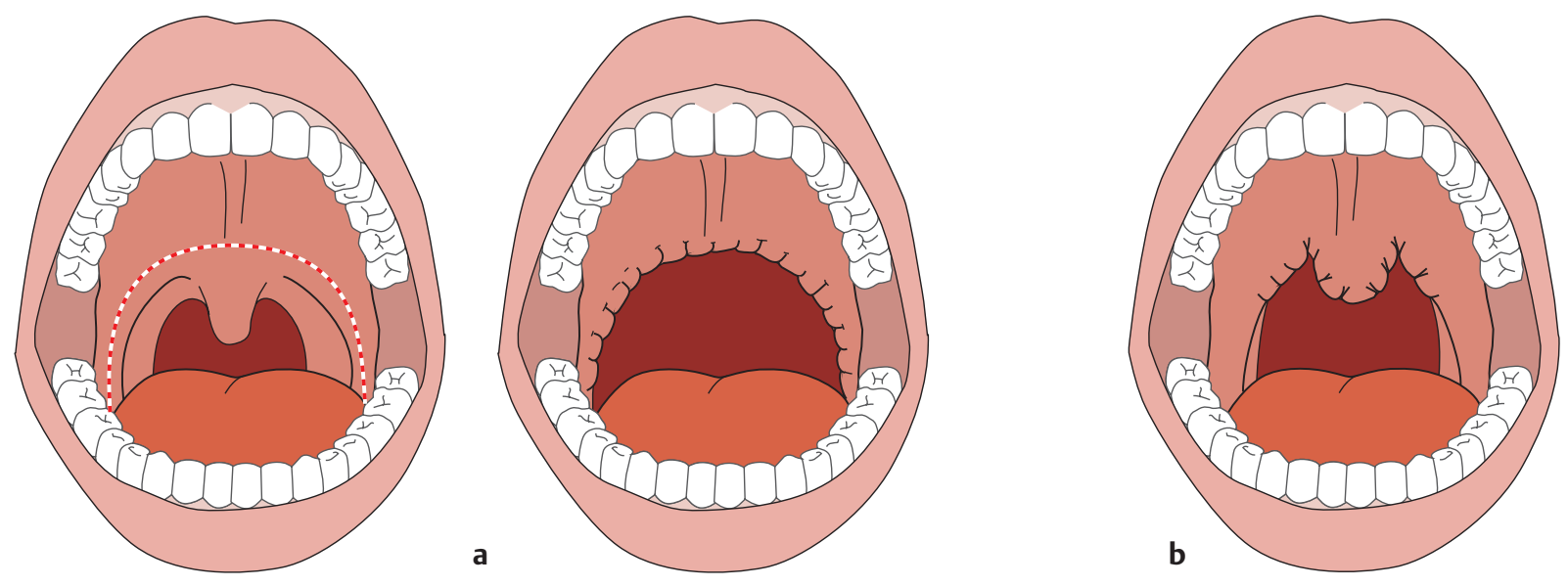

Abb. 14 Prinzipien der UPPP (Uvulopalatopharyngoplastik): radikale Technik (a) gegenüber funktioneller Technik (b). 
Hyoidsuspension. Über eine Vorverlagerung des Zungenbeines wird der Zungengrund erweitert. Die Methode wird überwiegend bei Versagen der Beatmungstherapie in Kombination mit anderen Eingriffen empfohlen.

Teilresektion der Zunge. Relativ invasives Verfahren mit Resektion der Zungengrundmuskulatur und passagerer Tracheotomie oder bei entsprechender Hyperplasie als Resektion der Zungengrundtonsille. Beide Eingriffe erfordern eine strenge postoperative Überwachung und tragen das Risiko der Nachblutung und Verletzung von Geschmackpapillen.

Zungenligatur. Der Zungengrund wird versucht durch Ligaturen nach vorn zu ziehen. Die Befestigung erfolgt an der Mandibula. Insgesamt ist die Studienlage dünn und die Resultate mit denen der Radiofrequenztherapie vergleichbar. Für die Methode wird bei mildem und schweren OSA ein EBM-Empfehlungsgrad C vorgeschlagen.

\section{Kieferchirurgische Verfahren}

Bei Fehlstellungen des Ober- und Unterkiefers können grundsätzlich 2 Verfahren zur Korrektur des nach hinten versetzten Unterkiefers empfohlen werden, die maxillo-mandibuläre Umstellungsosteotomie (EBM-Empfehlungsgrad B) und die Distraktionsosteogenese (EBM-Empfehlungsgrad D). In beiden Fällen wird nach Durchtrennen des Unterkiefer- und Oberkieferknochens der gesamte Unterkiefer und damit der Zungengrund nach vorn verlagert. Neben der Tracheotomie gelten diese Methoden auch in den Langzeitbeobachtungen als die erfolgreichsten chirurgischen Eingriffe. Nachteilig ist der hohe technische Aufwand bei relativ hohen Komplikationsraten im Vergleich zu den interstitiellen und weichteilresektiven Verfahren (invasive Methoden).

\section{Multi-Level-Chirurgie}

Dieser Begriff verdeutlicht, dass beim OSA häufig in mehreren Etagen anatomische und funktionelle Engstellen vorliegen, so dass einzelne chirurgische Maßnahmen weniger Erfolg versprechend sind als die Kombination mehrere Eingriffe. Häufig kann dabei sogar mit geringerer Invasivität vorgegangen werden als bei einer ausgedehnten resektiven Einzelchirurgie.

Merke: Von Multi-Level-Chirurgie wird heute gesprochen, wenn mindestens ein Eingriff am Zungengrund mit mindestens einem Eingriff an Weichgaumen oder Tonsille kombiniert wird [7].

Man unterscheidet heute die minimal invasive Multi-Level-Chirurgie - eine Radiofrequenztherapie des Zungengrundes erfolgt in Kombination mit anderen Eingriffen - von der invasiven MultiLevel-Chirurgie - eine Tonsillektomie oder Resektion am Zungengrund erfolgt in Kombination mit anderen Verfahren. Beide Methoden haben ihre Domäne bei Versagen der Beatmungstherapie (EBM-Empfehlungsgrad).

\section{Larynxchirugie}

Selten tragen Malformationen des Larynx im Kindesalter (z.B. Floppy Epiglottis) zur einem OSAS bei und können bei Versagen konservativer Maßnahmen mittels Laserteilresektionen oder Inzisionen erfolgreich behandelt werden (EBM-Empfehlungsgrad D)

\section{Tracheotomie}

Die Tracheotomie ist die erfolgreichste - wenngleich für den Patienten unbefriedigendste - chirurgische Methode zur Behandlung der OSA. Durch Studien gut belegt (EBM-Empfehlungsgrad A) wird sie bei schweren kardiovaskulären Begleiterkrankungen, Adipositas per magna und Versagen aller anderen Methoden nach insgesamt strenger Indikationsstellung angewandt.

\section{Zentrale Schlafapnoe-Syndrome}

Zentrale Schlafapnoe-Syndrome zeichnen sich durch eine Störung der zentralnervösen Innervation der Atemmuskulatur - also des zentralen Atemantriebs aus.

Merke: Bei zentraler Schlafapnoe besteht kein regulärer Atemfluss trotz offener Atemwege und es fehlt somit die für Obstruktionen typische respiratorische Atemanstrengung. Schnarchen kann dagegen vorliegen.

Die zentralnervösen Atemregulationsstörungen mit Instabilität der Atemkontrolle führen in der Regel zu einem vermehrten Abatmen von $\mathrm{CO}_{2}$ unter die Apnoeschwelle und anschließender Atempause. Erst bei Wiederanstieg des $\mathrm{CO}_{2}$ im Blut kommt es zum Wiedereinsetzen der Atmung mit entsprechender Weckreaktion und Fragmentierung des Schlafes. Klinisches Kennzeichen sind Atemstillstände infolge ausbleibender Atembewegung. Ein physiologisches Beispiel für eine zentrale Schlafapnoe ist die Regulation der Atmung unter hypoxischen Bedingungen (s. Höhenatmung). Unter den schlafbezogenen Atmungsstörungen werden die zentralen Schlafapnoe-Syndrome mit einer Häufigkeit zwischen 4 und 7\% angegeben [13, 14]. Man unterscheidet primäre von sekundären Formen ( $\bullet$ siehe Infobox). 
Zentrale Schlafapnoe-Syndrome

primäre Formen

- primär zentrale Schlafapnoe

- primäre Schlafapnoe im Säuglingsalter

sekundäre Formen

- im Rahmen von organischen Erkrankungen

- Cheyne-Stokes-Atmung

- bei Drogenabusus, Medikamentennebenwirkung

- periodische Atmung in großer Höhe

\section{Primäre Formen}

$\nabla$

Von primär zentraler Schlafapnoe wird gesprochen, wenn keine andere Ursache für die zentrale Apnoe gefunden werden kann. Modellhaft wird angenommen, dass eine erhöhte $\mathrm{CO}_{2}$-Rezeptorempfindlichkeit vorliegt, die zu einem periodischen Atemmuster mit zentralen Apnoen führt [13]. Die primäre Form des Erwachsenen ist insgesamt selten, tritt im mittleren Lebensalter auf und ist als idiopathisch zu betrachten. Klinische Zeichen sind ein wiederholtes nächtliches Erwachen infolge von 5 oder mehr Atemstillständen (> 10 Sek.) pro Stunde Schlaf mit Atemnot. Weitere klinische Folgen sind Tagesschläfrigkeit und Insomnie.

Die primäre Form des Säuglingsalters kann bei Frühgeborenen Ausdruck einer entwicklungsphysiologischen Unreife der zentralen Atmungskontrolle sein. Auch bei reifen Neugeborenen können innerhalb der ersten 6 Monate irreguläre Atemmuster mit Apnoephasen über 10 Sekunden ohne Krankheitswert auftreten und sind als Ausdruck der Unreife des ZNS zu betrachten [13]. Die klinische Relevanz ist umstritten und von der Länge der Atempausen, der Häufigkeit und der Auswirkung auf das Herz- Kreislaufsystem zu interpretieren. Im späteren Kindesalter können zentrale Schlafapnoen infolge von Infekten, metabolischen Störungen, Anämie, Reflux, Temperaturinstabilität, intrakraniellen Druckveränderungen oder medikamentenbedingt (Narkose) sekundär ausgelöst werden.

Diagnostisch erfordert die primär zentrale Form die Polysomnografie mit den Kennzeichen:

> kein Atemfluss

- kein Atemantrieb

- keine muskuläre Atmungsaktivität

bei Ausschluss jeglicher anderen die Symptomatik erklärbaren Erkrankung.

\section{Sekundäre Formen}

$\nabla$

\section{Cheyne-Stokes-Atmung}

Merke: Die häufigste Form zentraler Schlafapnoen ist die Cheyne-Stokes-Atmung.
Sie tritt mit einer Prävalenz von $25-40 \%$ bei Patienten mit Herzinsuffizienz, $10 \%$ bei Schlaganfall und weiterhin bei Niereninsuffizienz auf [5]. Charakteristisch ist dabei ein Atemmuster mit mindestens 10 Apnoen/Hypopnoen, die einen charakteristischen Crescendo-Decrescendo-Charakter der Atemkurve aufweisen. Die Diagnostik erfolgt mittels Polysomnografie, wobei eine Abgrenzung zum obstruktiven Schlafapnoe-Syndrom mittels Atemflussmessung und Messung der Atemanstrengung gelingt. Darüber hinaus gibtesjedochauchsekundärezentraleSchlafapnoen bei anderen schweren Erkrankungen (Hirnstamminfarkt), die ohne Cheyne-Stokes-Atmung einhergehen.

\section{Periodische Atmung in großer Höhe}

Die zentrale Schlafapnoe bei höhenbedingter periodischer Atmung tritt ab 4000m über NN auf und ist durch Müdigkeit, Erschöpfung und Luftnot gekennzeichnet. Im Schlaf kommt es zu periodisch auftretenden Apnoen infolge eines fehlenden Atemantriebes. Ursache ist eine physiologische Destabilisierung der Atemregulation. Aufgrund des in der Höhe niedrigeren Sauerstoffpartialdruckes muss, um eine ausreichende Sauerstoffsättigung im Blut zu erreichen, hyperventiliert werden. Im Schlaf kommt es dabei zu einer übermäßigen Abatmung von $\mathrm{CO}_{2}$. Da die Atemfrequenz über den $\mathrm{CO}_{2}$-Partialdruck gesteuert wird, entstehen regelmäßige zentrale Apnoephasen. Diese werden bei entsprechendem Anstieg des $\mathrm{CO}_{2}$-Partialdruckes mit dem Wiedereinsetzen der Atmung beantwortet. In der Regel erfolgt nach einigen Tagen die Anpassung der Apnoeschwelle, so dass die Symptomatik verschwindet.

\section{Medikamente, Drogen}

Zentrale Schlafapnoen aufgrund von Medikamenten und Drogen kommen beispielsweise bei Einnahme von Opioiden, Methadon und Morphin vor.

\section{Therapie zentraler Schlafapnoe- Syndrome \\ $\nabla$}

Die Therapie der primär zentralen SchlafapnoeSyndrome besteht in nichtinvasiven oder invasiven Beatmungsverfahren. 30\% der Patienten können mit einer CPAP-Therapie erfolgreich versorgt werden. Bei Versagen kommt eine Bi-LevelTherapie in Frage. Darüber hinaus werden in 20\% der Fälle Spontanremissionen beobachtet [5]. Bei der sekundären Form steht die Behandlung der Grunderkrankung bzw. der Ursache (z.B. des Medikamentenabusus) im Vordergrund. 


\section{Zentral-obstruktive Mischformen}

Obwohl der Begriff des gemischten SchlafapnoeSyndroms in der Klassifikation der ISCD 2 nicht vertreten ist, gibt es besonders beim obstruktiven Schlafapnoesyndrom zusätzlich zentrale Komponenten. Erkennbar werden die Mischformen häufig erst, wenn nach Beseitigung der obstruktiven Komponente im Schlaflabor (z.B. nach CPAP-Therapie) weiterhin zentrale Apnoephasen fortbestehen [5]. Diese Krankheitsbilder werden auch als komplexe Schlafapnoe bezeichnet.

\section{Schlafbezogene Hypoventilations-| Hypoxiesyndrome}

Bei diesen Syndromen entstehen während des Schlafes längere Phasen mit eingeschränkter Ventilation, obwohl der Atemantrieb intakt ist und keine Obstruktion der oberen Luftwege besteht. Als Ursachen kommen Störungen der $\mathrm{CO}_{2}$-Empfindlichkeit (Regelstörung) oder der Pump-Funktion der Lunge (Atempumpe) in Frage. Es wird bei Letzteren zwischen Erkrankungen mit Schwächen der Atemmuskulatur oder des neuromuskulären Komplexes (primäre Atempumpenschwäche) oder Erkrankungen mit pulmonalen Obstruktionen, Perfussionsstörungen des Lungeparenchyms, Thoraxdeformitäten und Adipositas (Erschöpfung der Atempumpe) unterschieden [5]. Das Syndrom kann aber auch ohne erkennbare Ursache kongenital oder beim Erwachsenen idiopathisch auftreten.

Demnach unterscheidet man folgende schlafbezogene Hypoventilations- bzw. Hypoxiesyndrome:

- idiopathischer Natur (schlafbezogene idiopathische alveoläre Hypoventilation)

- kongenitales Hypoventilationssyndrom

- bei Lungenparenchym- oder Lungengefäßerkrankungen

- bei Obstruktion der unteren Atemwege (bronchiale Obstruktion)

- bei neuromuskulären Erkrankungen mit Beteiligung der Atemmuskulatur oder Thoraxwanderkrankungen.

Die Diagnostik erfolgt mittels Polysomnografie und Blutgasanalysen während des Schlafes. Um eine Lungenerkrankung auszuschließen, erfolgen ergänzend Lungenfunktionsprüfungen sowie Echokardiografie.

Für schlafbezogene Hypoventilations-/HypoxieSyndrome ist eine Hyperkapnie mit einem $\mathrm{CO}_{2}-$ Druck charakteristisch, der gegenüber dem

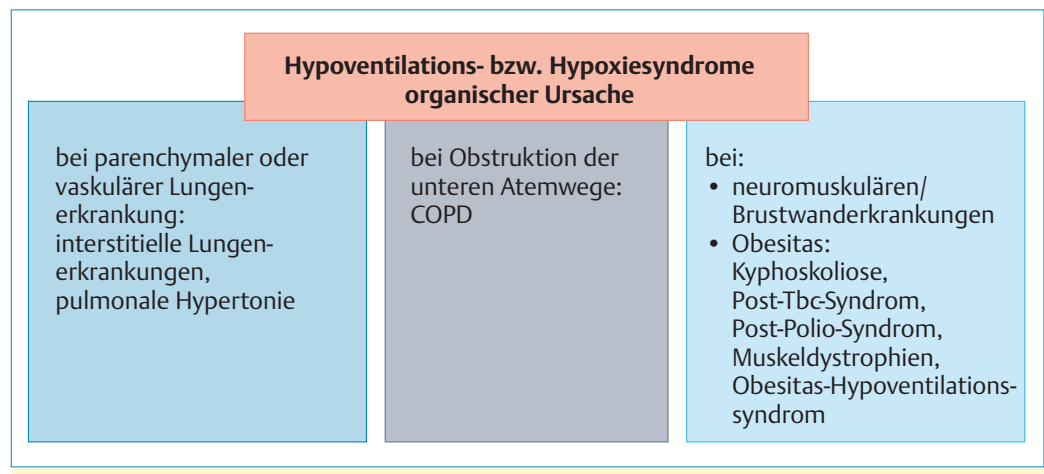

Abb. 15 Hypoventilations- und Hypoxiesyndrome organischer Ursache.

Wachzustand um mindestens $6 \mathrm{mmHg}$ erhöht ist und/oder einer Hypoxämie im Schlaf mit Sauerstoffentsättigungen unter $90 \%$ über mindestens $30 \%$ der Gesamtschlafzeit ohne dass der Atemantrieb verändert ist.

Die Syndrome treten selten idiopathisch auf. Häufiger dagegen sind die Hypoventilations-/ Hypoxämiesyndrome bei körperlichen Erkrankungen ( $\triangle$ Abb. 15). Die klinische Symptomatik ist dabei uncharakteristisch und von der Grunderkrankung überlagert. Durch die Ventilationsstörung besteht eine typische Belastungsdyspnoe bekleidet von Beinödemen infolge der Hyperkapnie und Kopfschmerzen [4]. Schlafbezogen sind Weckreaktionen mit Luftnot und konsekutiver Tagesschläfrigkeit die häufigsten subjektiven Symptome.

Die Therapie besteht in der Behandlung der Grunderkrankung. Bei unzureichendem Erfolg ist in der Regel eine nicht invasive Beatmungstechnik ausreichend.

\section{Zusammenfassung}

Schlafbezogene Atmungsstörungen sind vor allem aufgrund der kardiovaskulären Risiken aber auch der Vigilanzstörungen und deren Folgen von hoher klinischer und sozialer Bedeutung. Es lassen sich Störungen mit und ohne obstruktive Ursache unterscheiden. Das obstruktive Schlafapnoesyndrom des Kindes- und Erwachsenenalters lässt sich heute durch klinische und apparative Diagnostik sicher evaluieren. Goldstandard der Diagnostik ist dabei die Polysomnografie, die in schlafmedizinischen Zentren durchgeführt wird. Screeninguntersuchungen können bei bestimmten Voraussetzungen ambulant mittels Polygrafie durchgeführt werden. Hauptrisikofaktoren sind im Kindesalter die Hypertrophie der Gaumenund Rachenmandel und beim Erwachsenen die Adipositas. Während im Kindesalter die Domäne der Therapie des obstruktiven Schlafapnoesyndroms in der chirurgischen Behandlung liegt, ist der Therapieansatz im Erwachsenenalter nach 
der Ursache, Lokalisation und Prognose der Obstruktion individuell differenzierter zu betrachten. Die Beatmungstherapie wird vor allem für die Behandlung des schweren Schlafapnoesyndroms eingesetzt und dort ggf. durch chirurgische und verhaltenstherapeutische Maßnahmen sowie Hilfsmittel ergänzt. Bei gering- und mittelgradigem Schlafapnoesydrom kann die Behandlung durch diese Maßnahmen auch ohne Atemmaske erfolgreich sein. Neben dem obstruktiven Schlafapnoesynsdrom werden mit deutlich geringerer Prävalenz zentrale Schlafapnoesyndrome und schlafbezogene Hypoventilations-/ Hypoxiesyndrome unterschieden. Nach polysomnografischer Diagnostik werden diese Syndrome überwiegend über die Grunderkrankung bzw. mittels Beatmungstherapie behandelt.

\section{Über den Autor}

$\nabla$

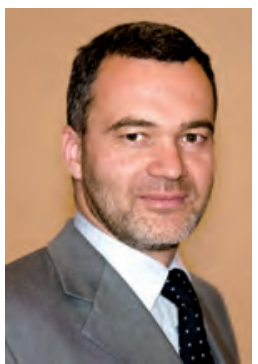

Thomas Zahnert

Jahrgang 1964, Univ.-Prof. Dr. med. habil. Dr. h.c. Direktor der Klinik und Poliklinik für Hals- Nasen- und Ohrenheilkunde, Medizinische Fakultät der Technischen Universität Dresden. 1984-1990 Medizinstudium an der Humboldt Universität Berlin und der Medizinischen Akademie Dresden. 1990 Wissenschaftlicher Mitarbeiter an der Medizinischen Fakultät der Technischen Universität Dresden, Klinik und Poliklinik für Hals-, Nasen- und Ohrenheilkunde. Aufbau eines Forschungslabors zur dynamischen Untersuchung der Mittelohrfunktion (Direktor: Prof. Dr. K.-B. Hüttenbrink). 1990 Promotion zum Thema Morphometrische Untersuchungen am Adenokarzinom des Dickdarms, Uniklinikum Dresden. 1995 Abschluss der Weiterbildung zum Facharzt für Hals-, Nasenund Ohrenheilkunde. 1995-2003 nationale und internationale Tutorentätigkeit als Cochlea-Implant- und Mittelohrchirurg. Erweiterung des Hörforschungslabors mit Aufbau eines Simulationsmodells in Kooperation mit dem Institut für Festkörpermechanik (Prof. Dr. H.-J. Hardtke, Prof. R. Schmidt). 2003 Habilitation: Laservibrometrische Untersuchungen zur Funktion des gesunden und rekonstruierten Mittelohres, Ernennung zum leitenden Oberarzt der Klinik für Hals-, Nasenund Ohrenheilkunde der med. Fakultät Dresden. 2004 Erster Vortragspreis für die beste Präsentation auf dem Europäischen HNO-Kongress. Verleihung der Ehrendoktorwürde durch die staatliche Universität Tblissi (Georgien). 2006 wiss. Vorträge auf Einladung in Chile und Argentinien sowie der Schweiz, wiss. Co-Organisation des internationalen Mittelohrworkshops „Middle Ear Mechanics in Research and Otosurgery" in Zürich. 2007 Ruferteilung auf
W3-Professuren-HNO an den Universitäten Magdeburg und Dresden. 2008 Gewinner Innovationswettbewerb Medizintechnik BMBF. 2010 Präsident der Vereinigung Mitteldeutscher HNOÄrzte.

\section{Korrespondenzadresse}

Prof. Dr. med. Dr. h.c. Thomas Zahnert Klinikdirektor

KI. u. PK f. HNO-Heilkunde

Universitätsklinikum Carl Gustav Carus an

der Technischen Universität Dresden

Fetscherstraße 74, 01307 Dresden

Deutschland

Tel: 0351/458-4420, Fax: 0351/458-4326

Email: Thomas.Zahnert@uniklinikum-dres-

den.de

Literatur

1 Poetz CF, Paditz E. Obstruktives Schlaf-Apnoe-Syndrom. Monatsschrift Kinderheilkunde 1998; 146: 826-836

2 Young T, Shahar E, Nieto FJ et al.; Sleep Heart Health Study Research Group. Predictors of sleepdisordered breathing in community-dwelling adults: the Sleep Heart Health Study. Arch Intern Med 2001; 162: 893900

3 Young T, Palta M, Dempsey J et al. The occurrenceof sleep-disordered breathing among middle-aged adults. N Engl J Med 1993; 328: 1230-1235

4 S3-Leitlinie - Nicht erholsamer Schlaf/Schlafstörungen. http://www.awmf.org/leitlinien/detail/11/063001.html. Zugriffsdatum 04.10.2011

5 Stuck B, Maurer JT, Schredl M, Weeß HG. Praxis der Schlafmedizin, 1. Aufl 2010; Heidelberg: Springer Verlag

6 Ross SD, Sheinhait IA, Harrison KJ et al. Systematic review and meta-analysis of the literature regarding the diagnosis of sleep apnea. Sleep 2000; 23: 519-32

7 Leitlinie - Therapie der obstruktiven Schlafapnoe des Erwachsenen. HNO 2009; 57: 1136-1156

8 Chan AS, Lee RW, Cistulli PA. Dental appliance treatment for obstructive sleep apnea. Chest. 2007; 132: 693-699

9 Ferguson KA, Ono T, Lowe AA, Keenan SP, Fleetham $J A$. A randomized crossover study of an oral appliance vs nasal-continuous positive airway pressure in the treatment of mild-moderate obstructive sleep apnea. Chest 1996; 109: 1269-1275

10 McArdle N, Dervereux G, Heidarnejad H et al. Longterm use of CPAP therapy for sleep apnea / hypopnea syndrome. Am J Respir Crit Care Med 1999; 159: 1108-1114

11 Dreher A et al. Das kindliche Schlafapnoe-Syndrom - Status quo in Diagnostik und Therapie. LaryngoRhino-Otol 2011; 90: 140-143

12 Verse T, Kroker B, Pirsig W, Brosch S. Tonsillectomy for treatment of obstructive sleep apnea in adults with tonsillar hypertrophy. Laryngoscope 2000; 110: 1556-1559

13 Schäfer T, Schläfke ME et al. Zentrale Schlafapnoe Pathophysiologie, Relevanz, Diagnostik und Therapie. Somnologie 2008; 12: 190-204

14 Westhoff M. Häufigkeit zentraler Apnoen und prädiktive Faktoren für ihr Auftreten sowie die Persistenz unter CPAP-Therapie. Pneumologie 2006; 60: 326-327 


\section{CME-Fragen Schlafbezogene Atmungsstörungen}

\section{Welche Aussage ist falsch?}

A. Unter Apnoe wird ein Atemstillstand verstanden.

B. Die Hypopnoe ist durch eine Reduktion des Atemflusses gekennzeichnet.

C. Schnarchen zählt zu den schlafbezogenen Atmungsstörungen.

D. Die Diagnose eines primären, harmlosen Schnarchens lässt sich erst nach Ausschluss einer schlafbezogenen Atmungsstörungen stellen.

E. Apnoe und Hypopnoe können anhand der apparativen Schlafdiagnostik genauer beschrieben werden.

2 Welche Aussage trifft nicht zu? Folgen des obstruktiven Schlafapnoe-Syndroms können sein:

A. erhöhtes Risiko für Hypertonie

B. erhöhtes Risiko für Polyneuropathien

C. erhöhtes Rsiko für Schlaganfälle

D. erhöhtes Unfallrisiko im Straßenverkehr

E. erhöhtes Risiko für Herzinsuffizienz

3 Welche Aussage ist richtig? Die Prätest-Wahrscheinlichkeit für eine Schlafapnoe ist hoch, wenn unter anderem vorhanden ist:

A. erhöhter Speichelfluss

B. rapide Gewichtsabnahme

C. Migräneattacken

D. Einschlafstörungen

E. Adipositas

\section{Welche Antwort ist richtig?}

A. Die Polygrafie beeinhaltet eine ausführliche apparative schlafmedizinische Diagnostik.

B. Die Polygrafie beinhaltet die EEG-Aufzeichnung.

C. Die Polygrafie liefert in bis zu $30 \%$ der Fälle falsch negative Ergebnisse.

D. Die Polygrafie zeichnet respiratorische, kardiovaskuläre und akustische Parameter während des Schlafs auf.

E. Die Polygrafie kommt bei neurologischen, psychiatrischen und kardiovaskulären Erkrankungen zum Einsatz.
5 Welche Aussage ist falsch? Bei der Polysomnografie werden neben den Parametern der Polygrafie zusätzlich erfasst:
A. Schlaf-EEG
B. Elektrookulogramm
C. Langzeit-RR-Messung
D. Elektromyogramm von M. mentalis, M. submentalis und M. tibialis anterior
E. Videometrie

\section{Welche Aussage ist korrekt?}
A. Bei der Schlafhygiene spielt die Einhaltung der individuellen Schlafdauer nur eine unter geordnete Rolle, da sie sehr stark variiert.
B. Polygrafie und Polysomnografie geben keine Rück- schlüsse auf die Lageabhängigkeit einer Schlafapnoe.
C. Gewichtsreduktion ist nicht zwingend Teil der Therapie bei OSA, da die Erfolgaussichten nur moderat sind.
D. Regelmäßiger Alkoholgenuss und reichhaltige Mahlzeiten am Abend stören die Schlafrhythmik.
E. Übergewicht ist kein Hauptrisikofaktor für eine OSA.

\section{Welche Antwort ist falsch? Eine Behandlung mittels einer Unterkieferprotrusionsschiene scheint besonders geeignet bei}
A. rückenlageassoziierter OSA
B. OSAS in Zusammenhang mit adenoiden Vegetationen
C. geringem Würgereiz
D. Retrogenie
E. Zungengrundobstruktion

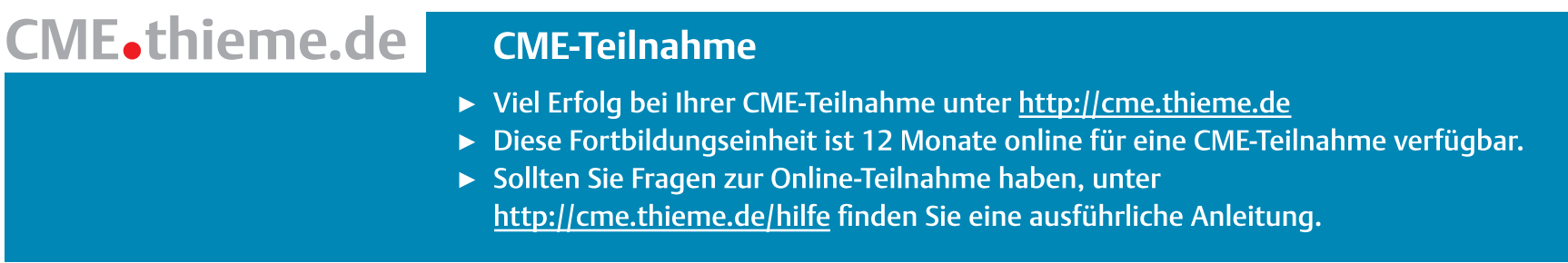


8 Welche Aussage zur nasalen Beatmungstherapie trifft zu?

A. Apnoen und Hypopnoen können zu etwa 50\% beseitigt werden.

B. Unter der Behandlung lässt sich der Blutdruck um ca. $20 \mathrm{mmHg}$ senken.

C. Je jünger die Patienten und je geringgradiger die Schlafapnoe, desto höher ist in der Regel die Maskentoleranz.

D. Das Risiko für Verkehrsunfälle läst sich nicht reduzieren.

E. Die nasale CPAP-Therapie ist der Gold-Standard für die Behandlung der mittel- bis schwergradigen OSA.

\section{Welche Aussage ist falsch?}

A. Korrekturen der Nasenatmung können sinnvoll sein, um die Maskenbeatmung zu erleichtern.

B. Eine alleinige Verbesserung der Nasenatmung durch chirurgische Korrektur kann zur Behandlung der OSA ausreichend sein.

C. Adenoide Vegetationen sind im Kindesalter zu 70-75\% der Fälle für eine OSA verantwortlich.

D. Die Tonsillotomie/Tonsillektomie hat auch im Erwachsenenalter eine gute Erfolgsrate bei der Behandlung der OSA.

E. Bei der Chirurgie des weichen Gaumens haben sich heute funktionserhaltende gegenüber radikalen Methoden durchgesetzt.
10 Welche Aussage zur zentralen Schlafapnoe ist korrekt?

A. Bei der zentralen Schlafapnoe besteht ein eingeschränkter Atemfluss bei verlegten Atemwegen.

B. Es kommt zur vermehrten $\mathrm{CO}_{2}$-Abatmung unter die Apnoeschwelle mit Atempausen.

C. Unter den schlafbezogenen Atemstörungen sind 15-20\% zentrale Schlafapnoe-Syndrome.

D. Die primäre Form tritt häufig erst im Erwachsenalter auf.

E. Die Therapie der primären Schlafapnoe-Syndrome besteht in vorrangig konservativen Methoden.

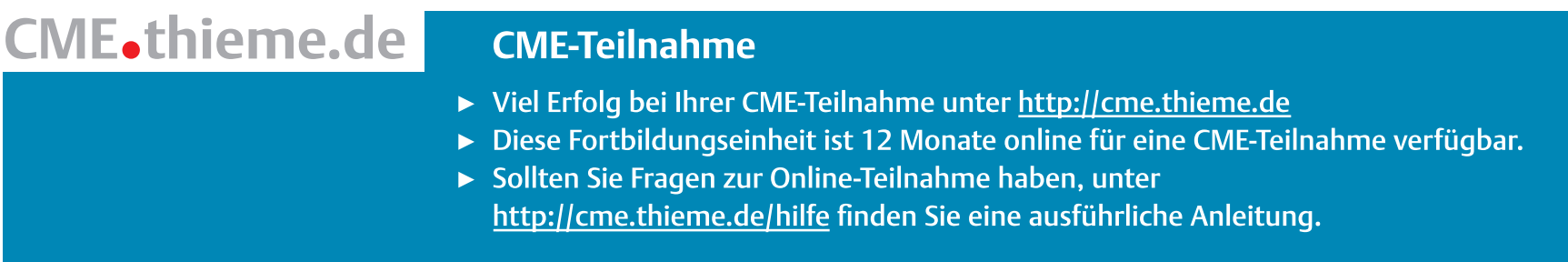

